\title{
Turning charge-density waves into Cooper pairs
}

\author{
Alla Chikina ${ }^{1,2,5}$, Alexander Fedorov ${ }^{1,3,5}$, Dilipkumar Bhoi $\mathbb{D}^{4}$, Vladimir Voroshnin ${ }^{3}$, Erik Haubold ${ }^{1}$, Yevhen Kushnirenko (iD) \\ Kee Hoon Kim (iD ${ }^{4}$ and Sergey Borisenko (iD ${ }^{1 \times}$
}

The relationship between charge-density waves (CDWs) and superconductivity is a long-standing debate. Often observed as neighbors in phase diagrams, it is still unclear whether they cooperate, compete, or simply coexist. Using angle-resolved photoemission spectroscopy, we demonstrate here that by tuning the energy position of the van Hove singularity in $\mathrm{Pd}$-doped $2 \mathrm{H}$ $\mathrm{TaSe}_{2}$, one is able to suppress CDW and enhance superconductivity by more than an order of magnitude. We argue that it is particular fermiology of the material that is responsible for each phenomenon, thus explaining their persistent proximity as phases.

npj Quantum Materials (2020)5:22; https://doi.org/10.1038/s41535-020-0225-5

\section{INTRODUCTION}

Two, perhaps most fascinating ordering phenomena in solid-state physics, superconductivity and charge-density waves (CDWs), have a lot in common. Both are symmetry breaking phases characterized by an energy gap in a single-particle spectrum and a complex order parameter representing the existence of electron-electron or electron-hole pairs. In both cases, a re-organization of charge into waves or pairs needs a mediator, and thus requires a strong interaction between electrons and another degree of freedom, for example, lattice vibrations ${ }^{1,2}$. Both often have precursor phases above the critical temperature, characterized by, for example, pseudogap and Fermi surface arcs ${ }^{3,4}$. Finally, both are remarkably well explained by BCS (Bardeen-Cooper-Schrieffer) theory in their simplest manifestations-conventional superconductors and 1D Peierls systems, where phonons and fermiology (Fermi surface, underlying dispersion, the density of states at the Fermi level) play an essential role ${ }^{5}$.

However, CDWs and superconductivity remain unexplained in more complicated cases, namely, in higher dimensions and high$T_{C}$ superconductors. Intriguingly, there both phases are often observed together, bordering in the generic phase diagrams. All hole-doped cuprates were found to exhibit $\mathrm{CDWs}^{6-8}$ and archetypal CDW-bearing quasi-2D transition metal dichalcogenides (TMDs) $2 \mathrm{H}-\mathrm{NbSe}_{2}$ and $2 \mathrm{H}-\mathrm{TaSe}_{2}$ are superconductors ${ }^{9,10}$. While it is clear that the conventional BCS theory cannot explain the high-temperature superconductivity in the cuprates, the debates whether the Fermi surface nesting, explaining Peierls transition, is a requisite for CDW in 2D or not are ongoing $3,4,11-20$.

The TMD's are very convenient systems to test whether the details of the electronic structure are important for the underlying mechanism of both phenomena, since the critical temperatures of both CDW and superconductivity are very different and can be relatively easily tuned. For example, crystallographically isostructural $2 \mathrm{H}-\mathrm{NbSe}_{2}$ and $2 \mathrm{H}-\mathrm{TaSe}_{2}$ exhibit transitions to incommensurate CDW (iCDW) state at $T_{\mathrm{iCDW}}=33$ and $120 \mathrm{~K}$, while the superconducting state is reached at $T_{C}=7.3$ and $0.133 \mathrm{~K}^{21,22}$, respectively, and the latter, in addition, enters a commensurate $\mathrm{CDW}(\mathrm{CCDW})$ state at $T_{\mathrm{CDW}}=90 \mathrm{~K}$. At the same time, the nesting of the Fermi surface in $2 \mathrm{H}-\mathrm{NbSe}_{2}$ was found to be weaker and a saddle-point singularity was found to be located below the chemical potential, contrary to $2 \mathrm{H}-\mathrm{TaSe}_{2}$, thus implying the crucial role of fermiology for both phenomena. Moreover, recent studies revealed that even moderate intercalation, application of pressure, or electron irradiation in one of the materials can lead to significant variation of the critical temperatures ${ }^{23-26}$. Since $\mathrm{Pd}$ intercalation induces more than an order of magnitude enhancement of $T_{C}$ in $2 \mathrm{H}-\mathrm{Pd}_{x} \mathrm{TaSe}_{2}{ }^{23}$ and the electronic structure of the pristine material is well known ${ }^{11-17,19,20}$, we initiated the present study to learn about the electronic structure of the Pd-intercalated one. Our measurements clearly identify a Lifshitz transition, which causes both destruction of nesting and shift of the van Hove singularity to the Fermi level, strongly implying that it is fermiology which plays an ultimate role in mechanisms of both ordering phenomena.

\section{RESULTS}

The electronic phase diagram of Pd-intercalated $2 \mathrm{H}-\mathrm{TaSe}_{2}$ is presented in Fig. $1 \mathrm{a}$, as summarized in ref. ${ }^{23}$. With increasing $\mathrm{Pd}$ concentration, the cCDW phase is suppressed dramatically compared to the iCDW phase. At the same time, the superconducting $T_{\mathrm{C}}(x)$ reveals a classic dome shape with an optimal $T_{\mathrm{C}}$ for $x=0.08-0.09$. Notably, the collapse of CCDW phase coincides with the doping level where an optimal $T_{C}$ is realized. In Fig. $1 \mathrm{~b}$, we have shown the temperature dependence of the in-plane resistivity, $\rho_{\mathrm{ab}}$, of $2 \mathrm{H}-\mathrm{Pd}_{x} \mathrm{TaSe}_{2}$ single crystal, close to the optimal $\mathrm{Pd}$ composition. For this composition, $\rho_{\mathrm{ab}}(\mathrm{T})$ does not show any signature of the cCDW transition (inset of Fig. $1 \mathrm{~b}$ ). $\rho_{\mathrm{ab}}$ starts to drop near the onset temperature, $T_{C}^{\text {on }} \sim 3.29 \mathrm{~K}$ and goes to zero at $T_{\mathrm{C}} \sim 3 \mathrm{~K}$. Figure $1 \mathrm{c}$ shows the temperature dependence of the diamagnetic susceptibility, $4 \pi \chi$, near the superconducting transition of the same crystal. The onset temperature of the diamagnetic susceptibility $(\sim 3 \mathrm{~K})$ determined from the zero-field cooled susceptibility curve agrees well with $T_{\mathrm{C}} \sim 3 \mathrm{~K}$, that is, zero resistivity temperature. At $1.9 \mathrm{~K}, 4 \pi \chi$ reaches to $93 \%$, indicating almost full Meissner shielding of the crystal used in the present study. Figure 1d displays the crystal structure and Brillouin zone of the $2 \mathrm{H}-\mathrm{TaSe}_{2}$.

Figure 1 implies that the CDW order hardly makes itself evident in the optimally intercalated samples. Neither resistivity nor

\footnotetext{
${ }^{1}$ IFW Dresden, P.O. Box 270116, 01171 Dresden, Germany. ${ }^{2}$ Department of Physics and Astronomy, Interdisciplinary Nanoscience Center, Aarhus University, 8000 Aarhus C, Denmark. ${ }^{3} \mathrm{Helmholtz-Zentrum} \mathrm{Berlin} \mathrm{für} \mathrm{Materialien} \mathrm{und} \mathrm{Energie,} \mathrm{Albert-Einstein-Strasse} \mathrm{15,} 12489$ Berlin, Germany. ${ }^{4}$ Department of Physics and Astronomy, Center for Novel States of Complex Materials Research and Institute of Applied Physics, Seoul National University, Seoul 151-747, Republic of Korea. ${ }^{5}$ These authors contributed equally: Alla Chikina, Alexander Fedorov. ${ }^{\circledR}$ email: s.borisenko@ifw-dresden.de
} 

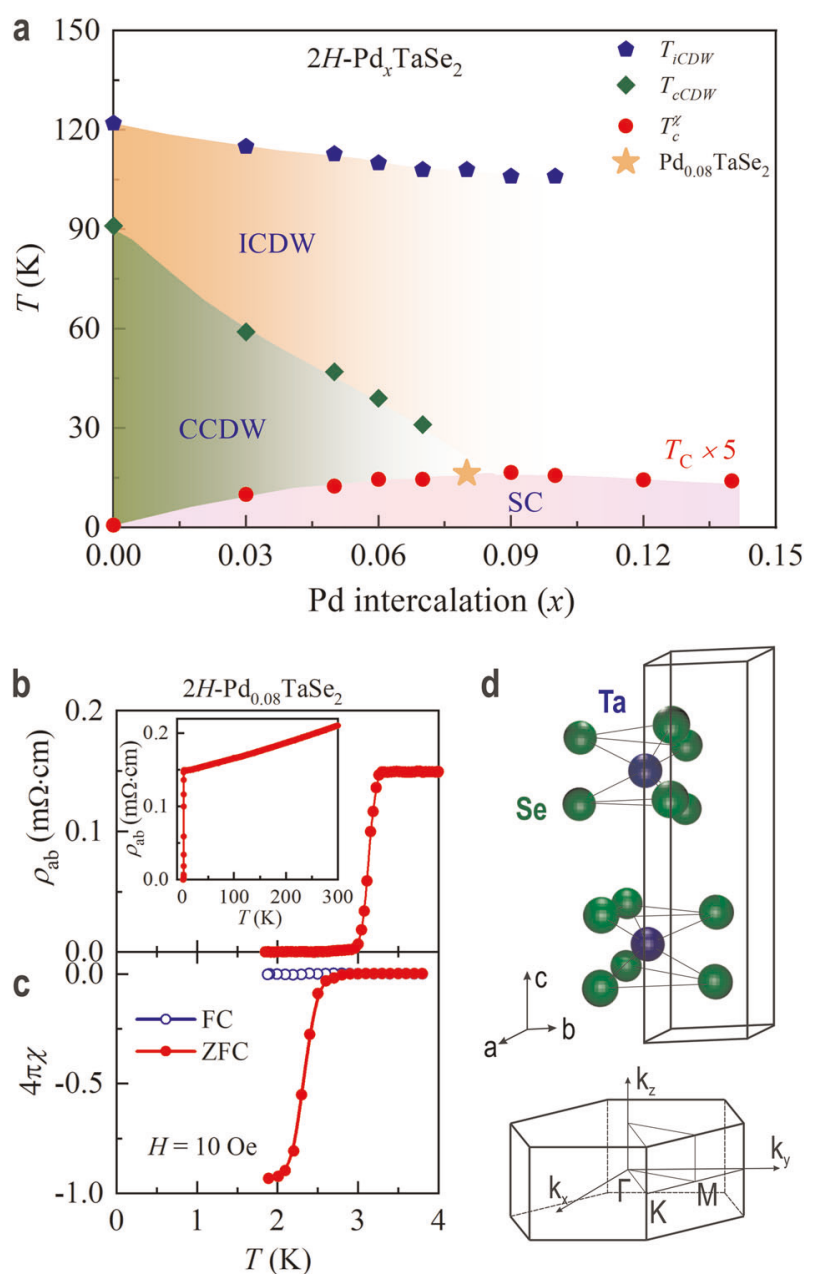

Fig. 1 Superconductivity and CDW in Pd-doped 2H-TaSe 2 . a The electronic phase diagram of $2 \mathrm{H}-\mathrm{Pd}_{x} \mathrm{TaSe}_{2}$ as summarized in ref. ${ }^{23}$. The orange star represents $2 \mathrm{H}-\mathrm{Pd}_{0.08} \mathrm{TaSe}_{2}$, close to the optimal doping, used in the present investigation. For clarity the superconducting transition temperatures, $T_{C}(x)$, have been multiplied by a factor of 5. $\mathbf{b}$ Temperature dependence of resistivity of $2 \mathrm{H}-\mathrm{Pd}_{x} \mathrm{TaSe}_{2}$ $(x=0.08)$ single crystal is presented below $4 \mathrm{~K}$ and its inset shows the same in a wide temperature window below 300K. c Magnetic susceptibility after zero-field cooling (ZFC, filled) and field cooling (FC, open symbols) near the superconducting transition. d Crystal structure and sketch of the Brillouin zone (space group P63/mmc) of the $2 \mathrm{H}-\mathrm{TaSe}_{2}$.

susceptibility or specific heat curves show the signature of the transition. In the pristine material, in contrast, the resistivity curve clearly marked the transition to the iCDW state at around $120 \mathrm{~K}^{23}$. Remarkably, the only spectroscopic change at this temperature was the opening of the pseudogap ${ }^{3}$ and no folding of the band structure occurred. Upon further lowering the temperature down to the commensurate lock-in transition, the replica of the bands started to appear and clear folding of the Fermi surface has been observed $^{3}$. Interestingly, nearly no thermodynamic change has been seen in $2 \mathrm{H}-\mathrm{TaSe}_{2}$ across this second transition.

In order to identify the possible spectroscopic signatures of the $\mathrm{CDW}$ transitions in $2 \mathrm{H}-\mathrm{Pd}_{0.08} \mathrm{TaSe}_{2}$, we recorded its Fermi surface map and show the results in Fig. 2a. The first and most crucial observation is that the Fermi surface contour does not show any evidence for the CDW state, neither for the commensurate nor for the incommensurate one. At a first glance, similar to hightemperature Fermi surface of $2 \mathrm{H}-\mathrm{TaSe}_{2}$, it consists of hole-like pockets centered at the $\bar{\Gamma}$ and $\overline{\mathrm{K}}$ points, and electron-like "dog- bones" around the $\overline{\mathrm{M}}$ point ${ }^{15}$. Earlier angle-resolved photoemission spectroscopy (ARPES) experiments have shown that the topology of $2 \mathrm{H}-\mathrm{TaSe}_{2}$ Fermi surface looks qualitatively similar in case of the high temperatures and the iCDW, while Fermi surface undergoes a $3 \times 3$ reconstruction at the cCDW state s, $^{3,16,20}$. In the commensurate phase, the "heads" of the "dog-bones" around the $\overline{\mathrm{M}}$ point are falling around the new $3 \times 3$ state $\bar{\Gamma}^{\prime}$ point and rounded triangles form splitting the "dog-bones" on doubly degenerate concentric pockets around new $\overline{\mathrm{K}}$ points. Here we do not see any of these changes. Also, no evidence for the pseudogap has been found in terms of spectral weight suppression or blurring of the parts of the Fermi surface. Therefore, we conclude that Pd doping suppresses all kinds of CDW order in optimally intercalated material.

The noticeable difference with the high-temperature Fermi surface of pristine sample ${ }^{3}$ are the larger "dog-bones" and their apparent touching in the point lying on $\bar{\Gamma}-\bar{K}$ high-symmetry direction. This results in a closure of the second Fermi surface sheet around the $\bar{\Gamma}$ and $\bar{K}$ points seen in Fig. $2 a$, that is, a topological Lifshitz transition from a set of electron-like Fermi surfaces to two hole-like ones. In order to clarify this and reveal further fine details of the band structure of $2 \mathrm{H}-\mathrm{Pd}_{0.08} \mathrm{TaSe}_{2}$, we present in Fig. $2 \mathrm{~b}-\mathrm{g}$ the data taken along the principal $\mathbf{k}$ paths of the Brillouin zone indicated by numbers and dashed lines in Fig. $2 a$.

Intensity distribution along $\bar{\Gamma}-\bar{K}$ (crossing 1 ) clearly shows the presence of several features, not just two, in contrast to the pristine material. In the former, one has always detected an electron-like dispersion with two crossings of the Fermi level, corresponding to the hole pockets around $\bar{\Gamma}$ and $\bar{K}$ points, respectively. In the present case, there is an additional intensity in between these crossings. The second derivative plot (Fig. 2d) and corresponding EDC (Fig. 2e) unambiguously demonstrate that there are electronic states in the immediate vicinity of the Fermi level. The only possible origin of these states is the energy shifted saddle point singularity, which remained unoccupied in the unintercalated material ${ }^{3}$. The data along the $\bar{\Gamma}-\bar{M}$ direction (crossings 2 and 3 ) clearly support our earlier conclusion as regards the size of the "dog-bone" Fermi surfaces along this highsymmetry direction: unlike in pristine $2 \mathrm{H}-\mathrm{TaSe}_{2}$, Fermi momenta are very similar, indicating the very small separation between the big hole pocket around $\bar{\Gamma}$ and heads of the "dog-bones". This explains the much stronger intensity of the former in Fig. 2a. It actually consists now of two Fermi surfaces with similar sizes. Finally, the cut 4-4 shown in Fig. 2f and corresponding second derivative plot (Fig. 2g) confirm all our previous observations related to the Fermi surface topology. Indeed, the electronic states between points 4 are located slightly below the Fermi level. The hole-like character of this, perpendicular to $\bar{\Gamma}-\bar{K}$ direction dispersion manifests the presence of the van Hove singularity of the saddle-point type in $2 \mathrm{H}-\mathrm{Pd}_{0.08} \mathrm{TaSe}_{2}$ and this feature is now located at the Fermi level or slightly below it (see Supplementary Fig. 1a, b).

Figure 3 schematically summarizes our findings and compares the low-energy electronic structures of three key materials: hightemperature phase of $2 \mathrm{H}-\mathrm{TaSe}_{2}, 2 \mathrm{H}-\mathrm{Pd}_{0.08} \mathrm{TaSe}_{2}$ and $2 \mathrm{H}-\mathrm{NbSe}_{2}$. As discussed above, in terms of the Fermi surface topology, we observe a gradual transformation of the electron-like "dog-bones" around $\bar{M}$ points into hole-like Fermi surface sheets around $\bar{\Gamma}$ and $\overline{\mathrm{K}}$ points. The reason for this transformation is the change of the energetic location of the van Hove singularity from above the Fermi level in $2 \mathrm{H}-\mathrm{TaSe}_{2}$ to below it in $2 \mathrm{H}-\mathrm{NbSe}_{2}$, which is illustrated in the lower panels of Fig. 3 by dashed dispersion with red arrow. Remarkably, in optimally intercalated $2 \mathrm{H}-\mathrm{Pd}_{0.08} \mathrm{TaSe}_{2}$ its bottom nearly coincides with the Fermi level. We have also shown that this singularity is indeed a saddle point (not band edge), since along the cut perpendicular to $\overline{\Gamma K}$, the dispersion is hole like (see cut 4-4 in Fig. 2 and Supplementary Fig. 1). The presence of the singularities in the vicinity of the Fermi level seems to be a 

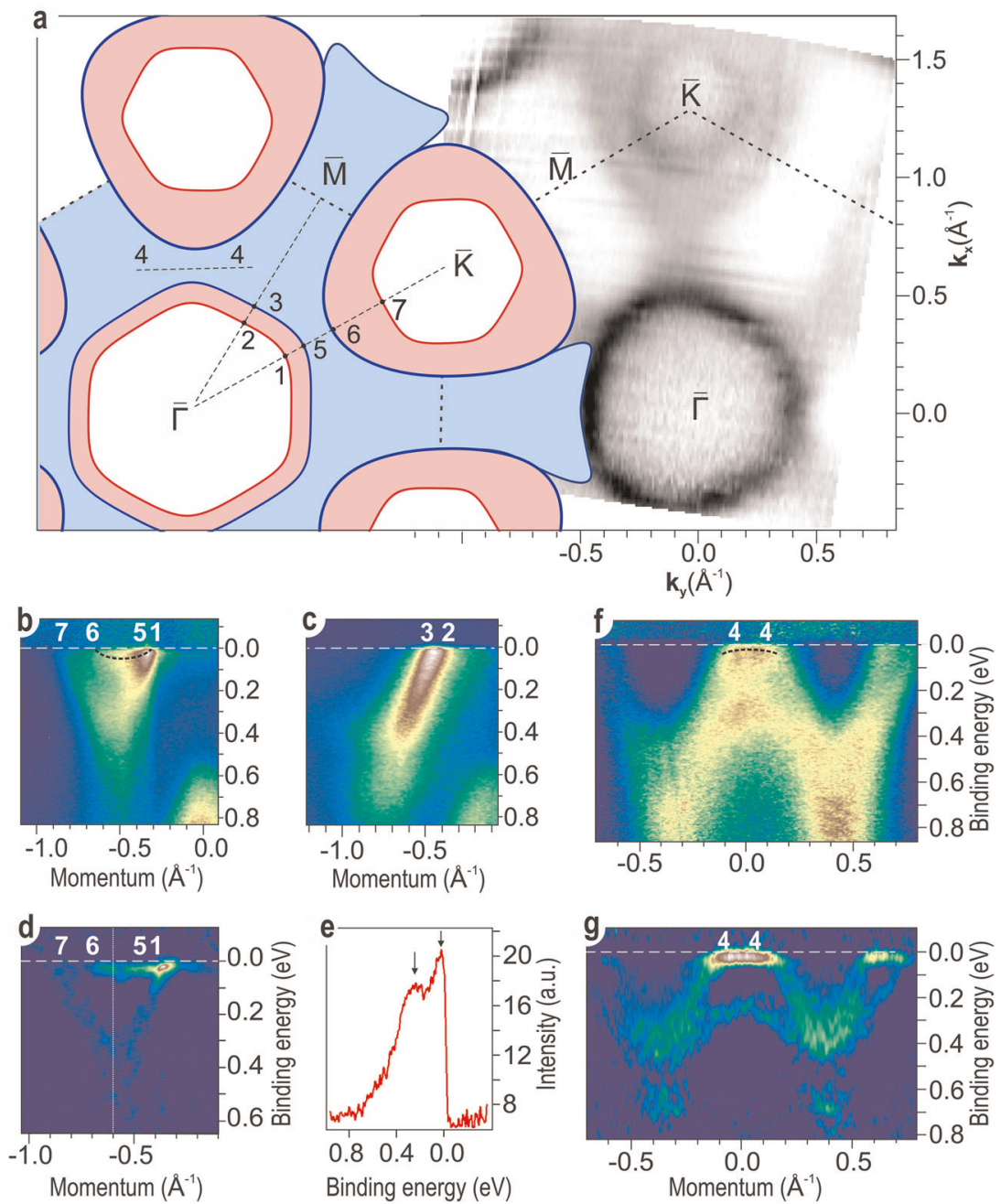

Fig. 2 Electronic band structure of $\mathbf{2 H}-\mathbf{P d}_{\mathbf{x}} \mathbf{T a S e}_{\mathbf{2}}$. a Fermi surface map taken at $80 \mathrm{eV}$ photon energy and a sketch of the Fermi surface pockets. $\mathbf{b}, \mathbf{c}, \mathbf{f}$ ARPES spectra along different momentum cuts in the BZ indicated in $\mathbf{a} . \mathbf{d}, \mathbf{g}$ Second derivative plots of intensity distributions from $\mathbf{b}, \mathbf{f}$, respectively. e EDC corresponding to the white vertical line in $\mathbf{d}$.

necessary requisite for superconductivity (see Fig. 2 in ref. ${ }^{27}$ ). The reason is known from $B C S$ theory ${ }^{2}$ : the strength of the particular singularity defines the density of states, and thus is directly related to the critical temperature. Intriguingly, all higher-temperature superconductors are characterized by stronger than usual (e.g., logarithmic divergence due to saddle points in 2D systems) singularities. The examples are the cuprates having the extended saddle-point singularities ${ }^{28}$ or iron-based superconductors having flat bands formed by multiband interactions (e.g., ref. ${ }^{29}$, Fig. 4 in ref. ${ }^{30}$, and ref. ${ }^{31}$ ). In this regard, we note the propensity to form an extended van Hove singularity in $2 \mathrm{H}-\mathrm{Pd}_{0.08} \mathrm{TaSe}_{2}$ visible in Fig. $2 d, g$, implying that further tuning of the electronic structure may result in still higher critical temperatures in this system. On the other hand, in the Bi2201 cuprates it seems that the strong van Hove singularity may also result in iCDW order outside the pseudogap region ${ }^{32}$.

Another result of our study seen in Fig. 3 is the overall bandwidth renormalization of the band structure. While the bands in intercalated sample seem to just shift down, which is natural because of electron doping (and this is the driving mechanism behind the relocation of the singularity), comparison with $2 \mathrm{H}$ $\mathrm{NbSe}_{2}$ shows that the bandwidth is drastically different ${ }^{33}$. While the electronic structures of $2 \mathrm{H}-\mathrm{Pd}_{0.08} \mathrm{TaSe}_{2}$ and $2 \mathrm{H}-\mathrm{NbSe}_{2}$ are qualitatively similar, the higher critical temperature in the latter may be explained either by higher density of states close to Fermi level, because of the overall stronger bandwidth renormalization or by better matching the energy of the pairing boson.

\section{DISCUSSION}

The evolution of the fermiology in mentioned dichalcogenides is in favor of nesting mechanism of CDW formation. A well known and very effective way to destroy the nesting is the shift of the chemical potential. Indeed, upon introducing additional charge carriers to the system, the sizes of $\bar{\Gamma}$ - and $\bar{K}$-centered hole-like Fermi surface sheets decrease simultaneously, quickly increasing the distance between their sections responsible for very good nesting in pristine material (see Fig. 1 and Supplementary Fig. A in ref. ${ }^{3}$ ). The other sections of the Fermi surface overlapping upon the shift by $\mathrm{CDW}$ vector in $2 \mathrm{H}-\mathrm{TaSe}_{2}$ are the parts of the $\overline{\mathrm{K}}$ barrel and electron-like "dog-bone." In this case, the distance between them decreases with electron doping destroying the nesting even more. As a result, we do not see any evidence for the CDW in this material. In $2 \mathrm{H}-\mathrm{NbSe}_{2}$, the change of the electronic structure is not due to doping and nesting is not destroyed completely. As a result, the CDW order survives only at significantly lower temperatures and remains incommensurate, making itself hardly visible spectroscopically ${ }^{4}$. We should point out that disorder induced by intercalated $\mathrm{Pd}$ atoms can be another reason for the suppression of CDW order in $2 \mathrm{H}-\mathrm{Pd}_{0.08} \mathrm{TaSe}_{2}{ }^{34}$. At the same time, 


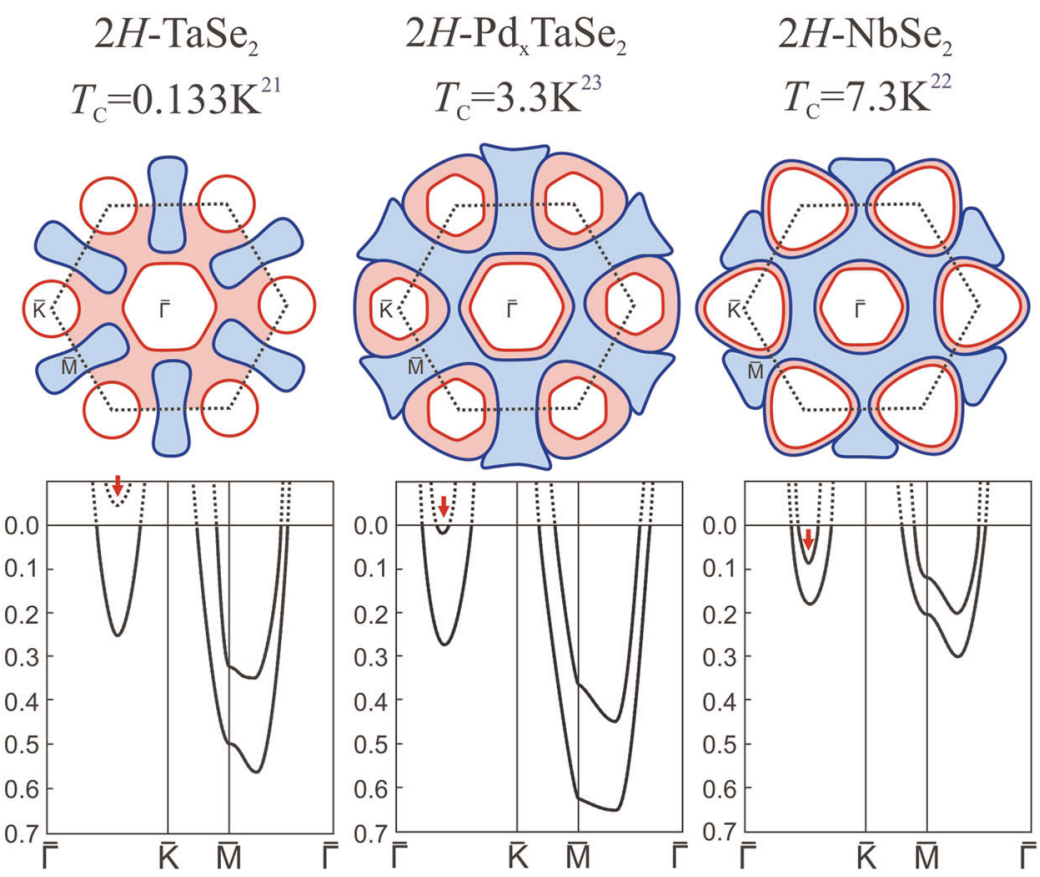

Fig. 3 Sketch of the electronic structure of $\mathbf{2 H}-\mathrm{TaSe}_{2}, \mathbf{2 H}-\mathbf{P d}_{\mathbf{0 . 0 8}} \mathrm{TaSe}_{2}$, and $\mathbf{2 H}-\mathrm{NbSe}_{2}$. Upper row: Sketch of the normal state Fermi surfaces. Lower row: Corresponding band structures. The sketches are the summary of multiple measurements aimed to determine the peaks of the spectral function from raw ARPES data.

the alternative mechanism of CDW formation based on the nesting of saddle points ${ }^{35}$ can most likely be ruled out: momentum separation of the unoccupied saddle points is very different from the CDW vectors in undoped case and even when they become occupied in the Pd-intercalated sample, CDW disappears.

Finally, our results shed more light on the coexistence of superconductivity and CDW in the phase diagrams. When the singularities are energetically close to the chemical potential, the resulting high density of states makes the system unstable with respect to the pairing. Already insignificant variation of the energy position of the singularity may quickly alter the electronic susceptibility, and thus nesting conditions because the Fermi velocities are very low. On the other hand, such variations may trigger the superconductivity, since the peaks of the density of states may reach the optimal position with respect to the Fermi level, for example, match with the low-energy boson. Obviously, the full picture of tuning the competition between the superconductivity and CDW should include the possible changes of such bosonic spectrum (e.g., phonons). Moreover, the role of the CDW fluctuations has to be clarified, since while the long-range ones definitely compete with superconductivity ${ }^{36}$, their quantum version may favor superconductivity ${ }^{37,38}$ and suppress long-range CDW order ${ }^{39}$.

Our study is in line with the recent observation of the stressdriven Lifshitz transition in $\mathrm{Sr}_{2} \mathrm{RuO}_{4}$, where the uniaxial pressure resulted in lowering of the saddle-point singularity below the Fermi level, which caused enhancement of the superconducting critical temperature ${ }^{40}$. We speculate that the same mechanism is responsible for the strong $T_{C}$ enhancement of $2 \mathrm{H}-\mathrm{TaSe}_{2}$ at high pressure ${ }^{25}$.

Although the full understanding of high- $T_{C}$ problem still requires a successful and comprehensive microscopic theory, we believe that the proximity of the van Hove singularities to the Fermi level is not only a necessary ingredient but also a crucial tuning parameter for controlling critical transition temperatures by pressure, strain, doping, intercalation, and so on.

\section{METHODS}

ARPES measurements were carried out at " 13 -ARPES" facility at BESSY at a temperature of $3 \mathrm{~K}$ within the range of photon energies $(50-80 \mathrm{eV})$ from the cleaved surface of high-quality single crystals. The overall energy and momentum resolutions were set to $\sim 8 \mathrm{meV}$ and $\sim 0.013 \AA^{-1}$, correspondingly. Polycrystalline samples of $\mathrm{Pd}_{x} \mathrm{TaSe}_{2}$ were synthesized via solid-state reaction method. Single crystal of the $\mathrm{Pd}_{x} \mathrm{TaSe}_{2}$ was grown by the chemical vapor transport method using $\mathrm{SeCl}_{4}$ as a transport agent. Millimeter-sized hexagonal-shaped crystals were collected from the cold end of the quartz tube. The phase purity of the samples was determined by the powder $\mathrm{x}$-ray diffraction using $\mathrm{Cu}$ Ka radiation at room temperature.

\section{DATA AVAILABILITY}

Data are available from the corresponding author upon reasonable request.

Received: 9 October 2019; Accepted: 12 March 2020;

Published online: 14 April 2020

\section{REFERENCES}

1. Grüner, G. Charge Density Waves in Solids (Addison-Wesley Co., Advanced Book Program, 1994).

2. Bardeen, J., Cooper, L. N. \& Schrieffer, J. R. Theory of superconductivity. Phys. Rev. 108, 1175-1204 (1957).

3. Borisenko, S. V. et al. Pseudogap and charge density waves in two dimensions. Phys. Rev. Lett. 100, 196402 (2008).

4. Borisenko, S. V. et al. Two energy gaps and Fermi-surface 'arcs' in $\mathrm{NbSe}_{2}$. Phys. Rev. Lett. 102, 166402 (2009).

5. Gabovich, A. M., Voitenko, A. I., Annett, J. F. \& Ausloos, M. Charge- and spindensity-wave superconductors. Supercond. Sci. Technol. 14, R1-R27 (2001).

6. Tranquada, J. M., Sternlieb, B. J., Axe, J. D., Nakamura, Y. \& Uchida, S. Evidence for stripe correlations of spins and holes in copper oxide superconductors. Nature 375, 561-563 (1995).

7. Ghiringhelli, $\mathrm{G}$. et al. Long-range incommensurate charge fluctuations in $(\mathrm{Y}, \mathrm{Nd})$ $\mathrm{Ba}_{2} \mathrm{Cu}_{3} \mathrm{O}_{6+x}$. Science 337, 821-825 (2012).

8. da Silva Neto, E. H. et al. Ubiquitous interplay between charge ordering and hightemperature superconductivity in cuprates. Science 343, 393-396 (2014). 
9. Moncton, D. E., Axe, J. D. \& DiSalvo, F. J. Study of superlattice formation in $2 \mathrm{H}-$ $\mathrm{NbSe}_{2}$ and $2 \mathrm{H}-\mathrm{TaSe}_{2}$ by neutron scattering. Phys. Rev. Lett. 34, 734-737 (1975).

10. Wilson, J. A., DiSalvo, F. J. \& Mahajan, S. Charge-density waves in metallic, layered, transition-metal dichalcogenides. Phys. Rev. Lett. 32, 882-885 (1974).

11. Liu, R., Tonjes, W. C., Greanya, V. A., Olson, C. G. \& Frindt, R. F. Fermi surface of $2 \mathrm{H}-$ $\mathrm{TaSe}_{2}$ and its relation to the charge-density wave. Phys. Rev. B 61, 5212-5216 (2000).

12. Valla, T. et al. Charge-density-wave-induced modifications to the quasiparticle self-energy in $2 \mathrm{H}-\mathrm{TaSe}_{2}$. Phys. Rev. Lett. 85, 4759-4762 (2000).

13. Rossnagel, K., Rotenberg, E., Koh, H., Smith, N. V. \& Kipp, L. Fermi surface, chargedensity-wave gap, and kinks in 2H-TaSe 2 . Phys. Rev. B 72, 121103 (2005).

14. Rossnagel, K. \& Smith, N. V. Spin-orbit splitting, Fermi surface topology, and charge-density-wave gapping in $2 \mathrm{H}-\mathrm{TaSe}_{2}$. Phys. Rev. B 76, 073102 (2007).

15. Inosov, D. S. et al. Fermi surface nesting in several transition metal dichalcogenides. N. J. Phys. 10, 125027 (2008).

16. Rossnagel, K. On the origin of charge-density waves in select layered transitionmetal dichalcogenides. J. Phys. 23, 213001 (2011).

17. Laverock, J. et al. k-resolved susceptibility function of $2 \mathrm{H}-\mathrm{TaSe}_{2}$ from angleresolved photoemission. Phys. Rev. B 88, 035108 (2013).

18. Rahn, D. J. et al. Gaps and kinks in the electronic structure of the superconductor $2 \mathrm{H}-\mathrm{NbSe}_{2}$ from angle-resolved photoemission at 1 K. Phys. Rev. B 85, 224532 (2012).

19. Ryu, H. et al. Persistent charge-density-wave order in single-layer $\mathrm{TaSe}_{2}$. Nano Lett. 18, 689-694 (2018).

20. Li, Y. W. et al. Folded superstructure and degeneracy-enhanced band gap in the weak-coupling charge density wave system 2H-TaSe 2 . Phys. Rev. B 97, 115118 (2018).

21. Kumakura, T., Tan, H., Handa, T., Morishita, M. \& Fukuyama, H. Charge density waves and superconductivity in 2H-TaSe . Czech. J. Phys. 46, 2611-2612 (1996).

22. Foner, S. \& McNiff, E. J. Upper critical fields of layered superconducting $\mathrm{NbSe}_{2}$ at low temperature. Phys. Lett. A 45, 429-430 (1973).

23. Bhoi, D. et al. Interplay of charge density wave and multiband superconductivity in $2 \mathrm{H}-\mathrm{Pd}_{x} \mathrm{TaSe}_{2}$. Sci. Rep. 6, 24068 (2016).

24. Chatterjee, U. et al. Emergence of coherence in the charge-density wave state of $2 \mathrm{H}-\mathrm{NbSe}_{2}$. Nat. Commun. 6, 6313 (2015)

25. Freitas, D. C. et al. Strong enhancement of superconductivity at high pressures within the charge-density-wave states of $2 \mathrm{H}-\mathrm{TaS}_{2}$ and $2 \mathrm{H}-\mathrm{TaSe}_{2}$. Phys. Rev. $B$ 93, 184512 (2016).

26. Cho, K. et al. Using controlled disorder to probe the interplay between charge order and superconductivity in $\mathrm{NbSe}_{2}$. Nat. Commun. 9, 2796 (2018).

27. Borisenko, S. Fewer atoms, more information. Nat. Mater. 12, 600-601 (2013).

28. Abrikosov, A. A., Campuzano, J. C. \& Gofron, K. Experimentally observed extended saddle point singularity in the energy spectrum of $\mathrm{YBa}_{2} \mathrm{Cu}_{3} \mathrm{O}_{6.9}$ and $\mathrm{YBa}_{2} \mathrm{Cu}_{4} \mathrm{O}_{8}$ and some of the consequences. Phys. C 14, 73-79 (1993).

29. Charnukha, A. et al. High-temperature superconductivity from fine-tuning of Fermi-surface singularities in iron oxypnictides. Sci. Rep. 5, 18273 (2015).

30. Maletz, J. et al. Photoemission and muon spin relaxation spectroscopy of the ironbased $\mathrm{Rb}_{0.77} \mathrm{Fe}_{1.61} \mathrm{Se}_{2}$ superconductor: Crucial role of the cigar-shaped Fermi surface. Phys. Rev. B 88, 134501 (2013).

31. Borisenko, S.V. et al. Strongly correlated superconductor with polytypic 3d dirac points. Preprint at https://arxiv.org/abs/1909.10806 (2019).

32. Peng, Y. Y. et al. Re-entrant charge order in overdoped $(\mathrm{Bi}, \mathrm{Pb})_{2.12} \mathrm{Sr}_{1.88} \mathrm{CuO}_{6+\delta}$ outside the pseudogap regime. Nat. Mater. 17, 697-702 (2018).

33. Borisenko, S. V. et al. Van Hove singularity as a possible origin of the bandwidth renormalization in layered superconductors. J. Phys. Chem. Solids 72, 562-564 (2011).

34. Nie, L., Tarjus, G. \& Kivelson, S. A. Quenched disorder and vestigial nematicity in the pseudogap regime of the cuprates. Proc. Natl. Acad. Sci. USA 111, 7980-7985 (2014).

35. Rice, T. M. \& Scott, G. K. New mechanism for a charge-density-wave instability. Phys. Rev. Lett. 35, 120-123 (1975).

36. Arpaia, R. et al. Dynamical charge density fluctuations pervading the phase diagram of a Cu-based high-T $\mathrm{T}_{\mathrm{c}}$ superconductor. Science 365, 906-910 (2018).
37. Grissonnanche, G. et al. Direct measurement of the upper critical field in cuprate superconductors. Nat. Commun. 5, 3580 (2014).

38. Caprara, S., Grilli, M., Lorenzana, J. \& Leridon, B. Doping-dependent competition between superconductivity and polycrystalline charge density waves. SciPost Phys. 8, 3 (2020)

39. Caprara, S., DiCastro, C., Seibold, G. \& Grilli, M. Dynamical charge density waves rule the phase diagram of cuprates. Phys. Rev. B 95, 224511 (2020).

40. Sunko, V. et al. Direct observation of a uniaxial stress-driven Lifshitz transition in $\mathrm{Sr}_{2} \mathrm{RuO}_{4}$. npj Quant. Mater. 4, 46 (2019).

\section{ACKNOWLEDGEMENTS}

We are grateful to Sabine Wurmehl for the fruitful discussions. This work is supported by DFG Grant Nos. BO1912/6-1, BO1912/7-1, and SPP1458, and BMBF Grant UKRATOP and VILLUM FONDEN through the Center of Excellence for Dirac Materials (Grant. No. 11744). The works at SNU have been supported by HYU-HPSTAR-CIS High Pressure Research Center (NRF-2016K1A4A3914691) and Korea Institute of Energy Technology Evaluation and Planning (20173010012940).

\section{AUTHOR CONTRIBUTIONS}

D.B. and K.H.K. provided and characterized high-quality single crystals. A.F., V.V., E.H and Y.K. carried out ARPES experiments. A.C. analyzed the data. A.C. and S.B. formulated the message and wrote the paper.

\section{COMPETING INTERESTS}

The authors declare no competing interests.

\section{ADDITIONAL INFORMATION}

Supplementary information is available for this paper at https://doi.org/10.1038/ s41535-020-0225-5.

Correspondence and requests for materials should be addressed to S.B.

Reprints and permission information is available at http://www.nature.com/ reprints

Publisher's note Springer Nature remains neutral with regard to jurisdictional claims in published maps and institutional affiliations.

Open Access This article is licensed under a Creative Commons Attribution 4.0 International License, which permits use, sharing, adaptation, distribution and reproduction in any medium or format, as long as you give appropriate credit to the original author(s) and the source, provide a link to the Creative Commons license, and indicate if changes were made. The images or other third party material in this article are included in the article's Creative Commons license, unless indicated otherwise in a credit line to the material. If material is not included in the article's Creative Commons license and your intended use is not permitted by statutory regulation or exceeds the permitted use, you will need to obtain permission directly from the copyright holder. To view a copy of this license, visit http://creativecommons. org/licenses/by/4.0/.

(c) The Author(s) 2020 